\title{
Long noncoding RNA expression profile and association with SLEDAI score in monocyte-derived dendritic cells from patients with systematic lupus erythematosus
}

Yilun Wang ${ }^{1+}$, Shuang Chen ${ }^{1+}$, Sunyi Chen ${ }^{1}$, Juan Du', Jinran Lin', Haihong Qin', Jie Wang ${ }^{2}$, Jun Liang ${ }^{1 *}$ and Jinhua $\mathrm{Xu}^{1 *}$

\begin{abstract}
Background: Monocyte-derived dendritic cells (moDCs) play important roles in the pathogenesis of systemic lupus erythematosus (SLE). Aberrant expression of long noncoding RNAs (IncRNAs) could affect the function of moDCs. The aim of this study was to explore the IncRNA expression profile in moDCs of SLE patients to provide new insights into SLE.

Methods: LnCRNA and mRNA microarrays were performed to identify differentially expressed IncRNAs and mRNAs in moDCs of SLE patients compared with normal controls. Bioinformatics analysis was also performed. Quantitative polymerase chain reaction (qPCR) was used to validate the results, and correlation analysis was used to analyze the relationship between these aberrantly expressed IncRNAs and SLE disease activity index (SLEDAI) scores.

Results: According to the gene expression profiles, 163 IncRNAs were differentially expressed between SLE and normal controls, including 118 that were upregulated and 45 that were downregulated. A total of $137 \mathrm{mRNAs}$ were differentially expressed in moDCs of patients with SLE, including 83 that were upregulated and 54 that were downregulated. Furthermore, qPCR data showed that IncRNA ENST00000604411.1 (18.23-fold, $P<0.001$ ) and ENST00000501122.2 (1.96-fold, $P<0.001)$ were upregulated and the other two IncRNAs, Inc-HSFY2-3:3 (0.42-fold, $P<0.001)$ and Inc-SERPINB9-1:2 (0.50-fold, $P=0.040)$, were downregulated in moDCs of SLE patients. The expression levels of ENST00000604411.1 $(r=0.593, P=0.020)$ and ENST00000501122.2 $(r=0.539, P=0.038)$ were positively correlated with the SLEDAI score, respectively.
\end{abstract}

Conclusions: The results indicate that the abnormal expression of IncRNAs in moDCs may be involved in the pathological processes of SLE. The expression level of ENST00000604411.1 and ENST00000501122.2 may have potential value for the assessment of disease activity in SLE.

Keywords: Systematic lupus erythematosus, Monocyte-derived dendritic cells, Long noncoding RNA, Expression profile

\footnotetext{
*Correspondence: Liangjun1976@medmail.com.cn; xjhhuashan@126.com

tYilun Wang and Shuang Chen contributed equally to this work.

${ }^{1}$ Department of Dermatology, Huashan Hospital, Fudan University, 12

Wulumuqi Zhong Road, Shanghai 200040, People's Republic of China

Full list of author information is available at the end of the article
}

(c) The Author(s). 2018 Open Access This article is distributed under the terms of the Creative Commons Attribution 4.0 International License (http://creativecommons.org/licenses/by/4.0/), which permits unrestricted use, distribution, and reproduction in any medium, provided you give appropriate credit to the original author(s) and the source, provide a link to the Creative Commons license, and indicate if changes were made. The Creative Commons Public Domain Dedication waiver (http://creativecommons.org/publicdomain/zero/1.0/) applies to the data made available in this article, unless otherwise stated. 


\section{Background}

Systematic lupus erythematosus (SLE) is an autoimmune disease that may damage multiple organs by autoantibodies and immune complexes. The precise etiology of SLE is still unclear and it might involve the regulation of genes, environments, and immune imbalance. Experimental evidence suggests that the pathogenesis of SLE is related to the failure of $\mathrm{T}$ - and $\mathrm{B}$-cell suppression mediated by defects in cell signaling, immune tolerance, and apoptotic mechanisms promoting autoimmunity [1].

Although $\mathrm{T}$ and $\mathrm{B}$ cells have been widely studied in SLE, the upper stream cells which could present autoantigens to them have only been emphasized more recently. Autoantigens are released mainly from secondary necrotic cells because of a defective clearance of apoptotic cells in patients with SLE [2]. Dendritic cells (DCs) are the most efficient antigen-presenting cells (APCs) in the human body. Recent research has associated lupus development with changes in the DC compartment, including altered DC subset frequency, localization, phenotype, and functional defects [3]. The dysfunction of DCs is related to the overreaction of $\mathrm{T}$ cells and $\mathrm{B}$ cells in SLE patients [4], including presenting autoantigens to autoreactive $\mathrm{T}$ cells, overproduction of proinflammatory cytokines and chemokines, suppression of Tregs, and promoting B cells to secret autoantibodies [5-8], which results in the loss of self-tolerance and production of autoantibodies.

Recent advances suggest that long noncoding RNAs (lncRNAs) regulate gene expression at the pretranscriptional, transcriptional, and post-transcriptional levels [9]. LncRNAs mediate their molecular functions through a multitude of mechanisms [10]. An lncRNA could interact with proteins in the cytoplasm as a guide, scaffold, or decoy molecule [10]. LncRNAs could also promote or repress the translation of mRNAs in the cytoplasm. For example, the antisense IncRNA BACE1-AS rapidly and reversibly upregulates BACE1 levels in response to a variety of stresses. Since BACE1 and BACE1-AS form an RNA duplex, the duplex may act to alter the secondary or tertiary structure of BACE1 and thereby increase its stability [11]. In the nucleus, IncRNAs can act in cis to control local allele-specific functions or in trans at one or more genomic loci to regulate gene expression.

LncRNAs are important in regulating the differentiation and function of DCs. Lnc-DC, which is exclusively expressed in human conventional DCs, can affect cellular differentiation (monocytes into dendritic cells) and reduce the capacity of DCs to stimulate T-cell activation by activating the transcription factor STAT3 [12]. The expression of the IncRNA HOTAIRM1 was downregulated when monocytes differentiated into DCs, and silencing of HOTAIRM1 caused changes in the expression of several monocyte differentiation markers such as CD14 and $\mathrm{B} 7 \mathrm{H} 2$ [13]. As a result, lncRNAs are able to cause clinical diseases involving the dysfunction of DCs. However, it is largely unknown whether lncRNAs participate in the pathogenesis of SLE by regulating the function of DCs; this is therefore the focus of this study.

\section{Methods \\ Subjects}

Fifteen female SLE patients were recruited from the inpatient service in Huashan Hospital, Fudan University. The diagnostic criteria were in accordance with the 1997 American College Rheumatology revised criteria for the classification of SLE. Relevant clinical and laboratory information regarding the patients is shown in Table 1 . Fifteen age-matched female healthy controls were also recruited. The study was approved by the Independent Ethics Committee of Huashan Hospital and written informed consent was obtained from all subjects. All the experiments were carried out in accordance with relevant guidelines and regulations of Huashan Hospital.

\section{Cell culture}

In-vitro differentiation of human monocytes into DCs was performed as described previously [8]. Briefly, peripheral blood mononuclear cells (PBMCs) were isolated and $\mathrm{CD} 14^{+}$monocytes were sorted by positive selection using magnetic beads (Miltenyi Biotec, Germany). The cells were then cultured for 5-7 days in RPMI 1640 supplemented with $1000 \mathrm{U} / \mathrm{ml}$ granulocyte/macrophage colony-stimulating factor (GM-CSF) and $1000 \mathrm{U} / \mathrm{ml}$ interleukin (IL)-4 (PeproTech, Rocky Hill). For monocyte-derived dendritic cell (moDC) maturation, $1 \mu \mathrm{g} / \mathrm{ml}$ lipopolysaccharide (LPS; Escherichia coli type 055:B6; Sigma) was added to the medium at day 6. The following antigens were used to identify the phenotype of moDCs: CD11c, HLA-DR, CD40, CD86, CD83, and

Table 1 Clinical and laboratory characteristics of the patients with SLE in the study

\begin{tabular}{ll}
\hline Characteristic & $\operatorname{SLE}(n=15)$ \\
\hline Sex, male/female $(n)$ & $0 / 15(15)$ \\
Age (years), median (range) & $33(17-57)$ \\
Duration (months), median (range) & $3(1-60)$ \\
SLEDAl score, median (range) & $15(10-30)$ \\
ANA > 1:320, yes/no ( $n)$ & $15 / 0(15)$ \\
Anti-dsDNA (IU/ml), median (range) & $391.9(25.9-800)$ \\
Hypocomplementemia, yes/no $(n)$ & $12 / 3(15)$ \\
Organ involvement, yes/no ( $n)$ & $6 / 9(15)$ \\
Steroids, yes/no $(n)$ & $9 / 6(15)$ \\
Immunosuppressive drugs, yes/no $(n)$ & $15 / 0(15)$ \\
\hline ANA antinur
\end{tabular}

ANA antinuclear antibody, SLE systemic lupus erythematosus, SLEDAl systemic lupus erythematosus disease activity index 
low expression of CD14. The antibodies were all bought from eBioscience (San Diego).

\section{LncRNA and mRNA microarray}

LncRNA expression profiling was determined by Human 4*180 K lncRNA arrays manufactured by Agilent Technologies (Santa Clara, CA), including 63,431 lncRNA probes and 39,887 mRNA probes. Each transcript was represented using $1-5$ probes to improve statistical confidence. The IncRNA and mRNA expression data have been deposited into the Gene Expression Omnibus (GEO) under accession number GSE89240.

\section{Gene function analysis}

The Database for Annotation, Visualization and Integrated Discovery (DAVID; https://david.ncifcrf.gov) was utilized to identify the molecular function represented in the gene profile. Furthermore, the Kyoto Encyclopedia of Genes and Genomes (KEGG) database (https:// www.genome.jp/kegg/) was also used to analyze the potential functions of these target genes in the pathways. The cut-off criterion for false discovery rate (FDR) was set at less than 0.05 .

\section{Coexpression network construction}

The IncRNA-mRNA coexpression network was constructed based on the correlation between the differentially expressed lncRNAs and mRNAs using Cytoscape. Pearson correlation analysis was used to evaluate the significance of the correlation between the expression levels between each pair of genes. Pearson correlation coefficients were selected for inclusion in the network when they were above 0.95 .

\section{Cis and trans target gene analysis of IncRNAs}

The gene location for different lncRNAs on the chromosome was determined. Subsequently, the common lncRNA coexpression genes were intersected to identify the genes $10 \mathrm{kbp}$ upstream or downstream of the lncRNAs as potential 'cis' genes. For the trans analyses, we predicted the trans-associated genes of the differentially expressed lncRNAs with RNAplex v0.2. The RNAplex parameters were set as e $\leq-30$ in the current study to identify the trans-associated genes, and genes that were found to be located on the same chromosome as the lncRNA were excluded.

\section{Quantitative real-time polymerase chain reaction (qRT- PCR)}

Total RNA was reverse transcribed using a PrimeScript RT reagent Kit with gDNA Eraser (Perfect Real Time; TaKaRa, Dalian, China) in accordance with the manufacturer's instructions. The expressions of selected lncRNAs were analyzed using qRT-PCR with an ABI Power SYBR
Green PCR Master Mix (ABI, USA). Glyceraldehyde 3-phosphate dehydrogenase (GAPDH) mRNA was used as an internal control. The primers are listed in Table 2 . For quantitative results, expression of each lncRNA was represented as a fold change using the $2^{-\Delta \Delta C t}$ method and then statistically analyzed.

\section{Statistics}

Continuous variables are expressed as means (SD) and categorical variables as frequencies (\%). The Student $t$ test or one-way analysis of variance was used to compare continuous variables. All $P$ values were estimated in a two-tailed fashion. Differences were considered to be statistically significant at $P<0.05$. Data were analyzed using SPSS 13.0 (SPSS Inc., IL, USA). The relationships between the expression levels of lncRNAs and systemic lupus erythematosus disease activity index (SLEDAI) were analyzed by Pearson's correlation coefficient.

\section{Results}

Differential expression profile of IncRNAs in moDCs of SLE

Dendritic cells were differentiated from $\mathrm{CD} 14^{+}$monocytes. MoDCs were identified using flow cytometry. The phenotype of moDCs displayed high expression of HLA-DR, CD83, CD11c, CD86, and CD40, and low expression of CD14 (Fig. 1). To profile differentially expressed lncRNAs in moDCs of patients with SLE, we performed a genome-wide analysis of IncRNA expression in moDCs between SLE patients and normal controls. Volcano plot analysis showed that $163(0.22 \%)$ of the IncRNAs were significantly differentially expressed in moDCs of patients with SLE (Fig. 2a), including 118 upregulated and 45 downregulated lncRNAs (fold change $\geq 2.0, P<0.05$ ) (Fig. $2 b$ ). We categorized these differentially expressed lncRNAs into six classes: intergenic, intronic sense, intronic antisense, bidirectional, exonic sense, and exonic antisense. We

Table 2 The sequences of quantitative polymerase chain reaction primers

\begin{tabular}{|c|c|}
\hline Primer name & Sequence (5' to $\left.3^{\prime}\right)$ \\
\hline gapdh(Hs) forward & TGACTTCAACAGCGACACCCA \\
\hline gapdh(Hs) reverse & CACCCTGTTGCTGTAGCCAAA \\
\hline ENST00000604411.1 forward & AGCCCCACTTCACATTAGACC \\
\hline ENST00000604411.1 reverse & TGATGTTGCAGTCCTGTGAGG \\
\hline ENST00000501122.2 forward & TTCTGCTITCTGCCCATGTA \\
\hline ENST00000501122.2 reverse & GTGGCAGTGACAACCTCTCA \\
\hline ENST00000568394.1 forward & TCTCTCCCCAGTGACAGTACA \\
\hline ENST00000568394.1 reverse & GCGATCTGTAGTCCAGGTGT \\
\hline Inc-HSFY2-3:3 forward & TGATTGGAAGATGGAAGTGGA \\
\hline Inc-HSFY2-3:3 reverse & GCTCCACCTGAAACTCATTTG \\
\hline Inc-SERPINB9-1:2 forward & GGGGAAAATAAAAGGGATGGT \\
\hline Inc-SERPINB9-1:2 reverse & TCTCTCTTAGGGAAGGGCATT \\
\hline
\end{tabular}



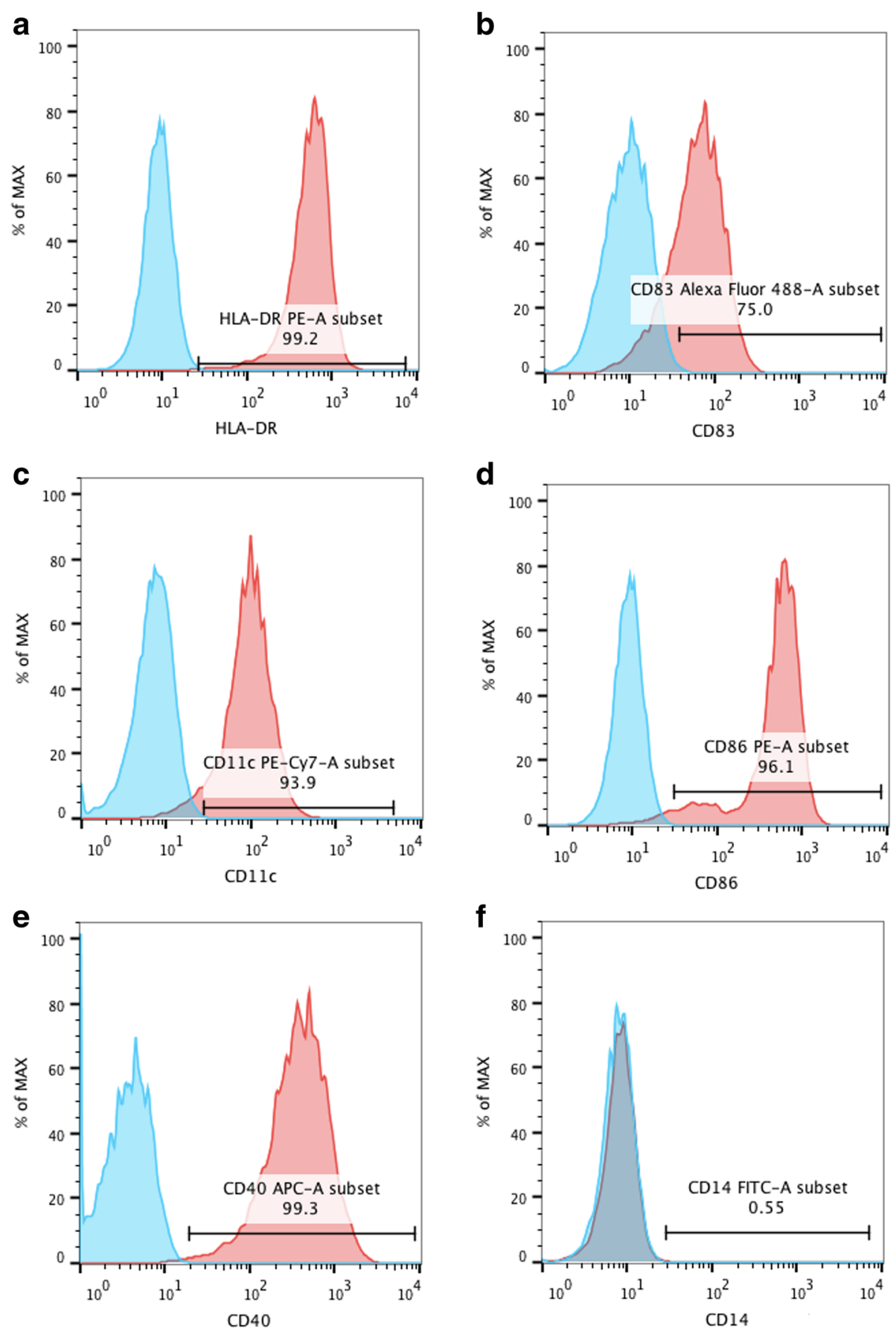

Fig. 1 moDCs were identified using flow cytometry. The phenotype of the cells displayed high expression of a HLA-DR, b CD83, c CD11C, d CD86, and $\mathbf{e}$ CD40, and $\mathbf{f}$ low expression of CD14

observed 150 intergenic, 2 intronic sense, 1 intronic antisense, 5 bidirectional, 4 exonic sense, and 1 exonic antisense lncRNA (Additional file 1: Table S1).

Differential expression profile of mRNAs in moDCs of SLE A total of $137(0.34 \%)$ mRNAs were differentially expressed in moDCs of patients with SLE compared with normal controls (fold change $\geq 2.0$, $P<0.05$ ) (Fig. 2c). Among them, 83 mRNAs were upregulated and 54 mRNAs were downregulated (Fig. 2d, Additional file 2: Table S2). Gene ontology (GO) analysis was performed to classify these differentially expressed mRNAs into three domains: biological process, molecular function, and 

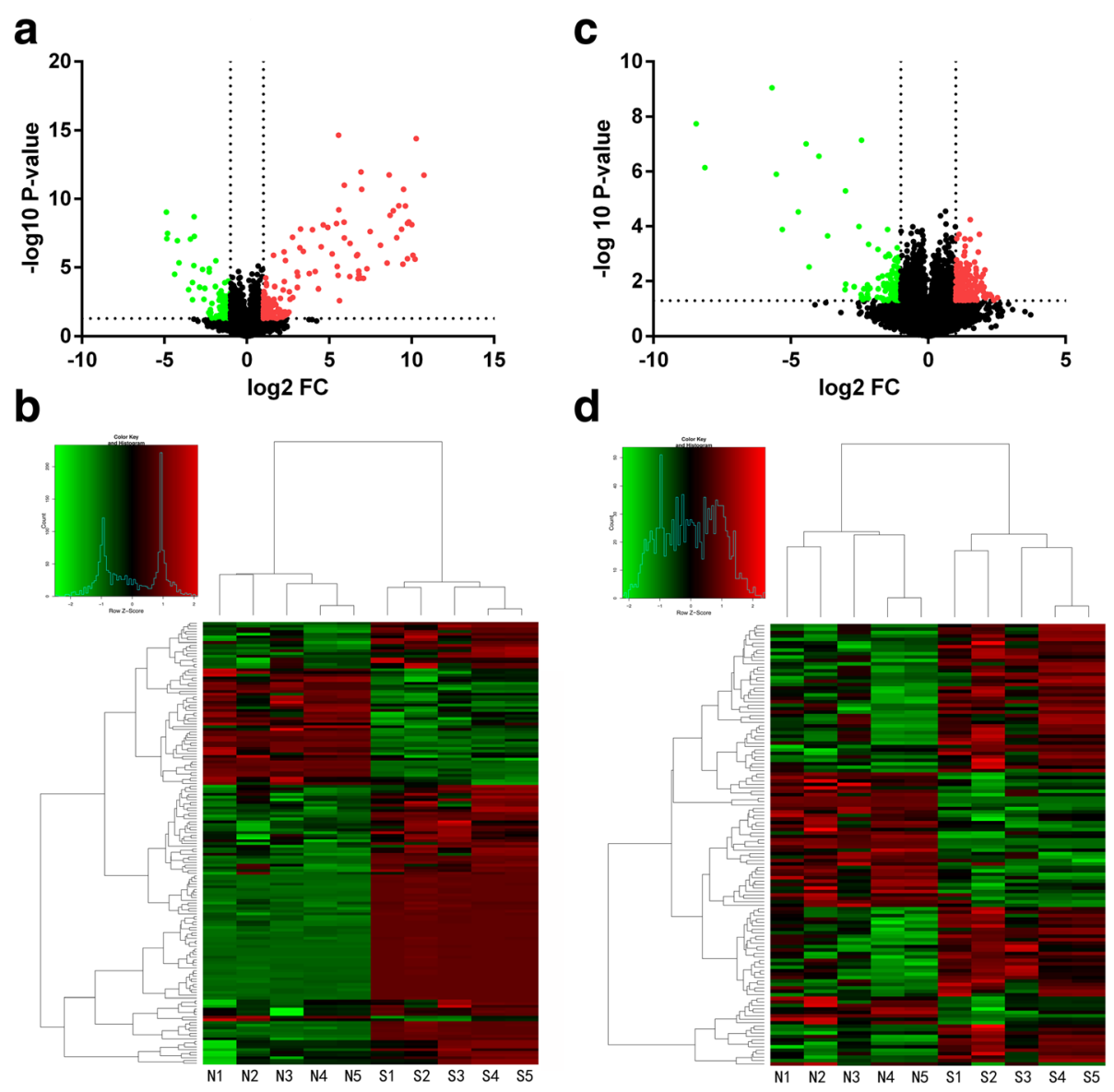

Fig. 2 Volcano plots and hierarchical clusters of differentially expressed IncRNAs and mRNAs. a LncRNA volcano plots of SLE patients versus normal controls. b A total of 163 IncRNAs were differentially expressed in moDCs of patients with SLE $(n=5)$ compared with normal controls $(n=5)$, including 118 upregulated and 45 downregulated IncRNA (fold change $(F C) \geq 2.0, P<0.05$ ). c mRNA volcano plots of SLE patients versus normal controls. $\mathbf{d}$ A total of 137 mRNAs were differentially expressed in moDCs of patients with SLE $(n=5)$ compared with normal controls $(n=5)$, including 83 upregulated and 54 downregulated mRNAs (fold change $\geq 2.0, P<0.05$ ). In $\mathbf{a}$ and $\mathbf{c}$, each point represents a different transcript. Red points represent genes that were significantly upregulated and green points represent genes that were significantly downregulated. In $\mathbf{b}$ and $\mathbf{d}$, relatively high expression is indicated by red shading and relatively low expression is indicated by green shading. N1-5 represents normal controls and S1-5 represents the patients with SLE

cellular component. In the biological process domain, the GO terms for the differentially expressed mRNAs included cell migration, cell-cell adhesion, and T-cell costimulation, etc. (Fig. 3a). In the cellular component domain, the top five GO terms were extracellular space, protein complex, transcription factor complex, endoplasmic reticulum lumen, and extracellular matrix (Fig. 3b). In the molecular function domain, the GO terms contained signal transducer activity, chemokine activity, and ubiquitin binding (Fig. 3c). KEGG pathway analysis was also conducted to identify the key signaling pathways and the relationships among the differentially expressed mRNAs. We identified 19 signaling pathways that were enriched in moDCs of SLE. The top five pathways were PI3K-Akt signaling pathway, cytokine-cytokine receptor interaction, protein digestion and absorption, extracellular matrix-receptor interaction, and hematopoietic cell lineage (Fig. 3d).

\section{Construction of the coexpression network with differentially expressed IncRNAs and mRNAs}

Next, we constructed a coexpression network of these coding-noncoding genes that included the differentially expressed lncRNAs and mRNAs. Our data showed that the coexpression network was composed of 127 network nodes and 316 connections between 62 lncRNAs and 65 mRNAs. This coexpression network indicated that one lncRNA could target, at most, 16 coding genes and that one coding gene could correlate with at most 12 lncRNAs (Additional file 3: Figure S1). NR_024243 and lnc-BSPH1-2:1 were the most connected lncRNAs. NMT1, NPM3, and UPB1 were the most connected mRNAs. 


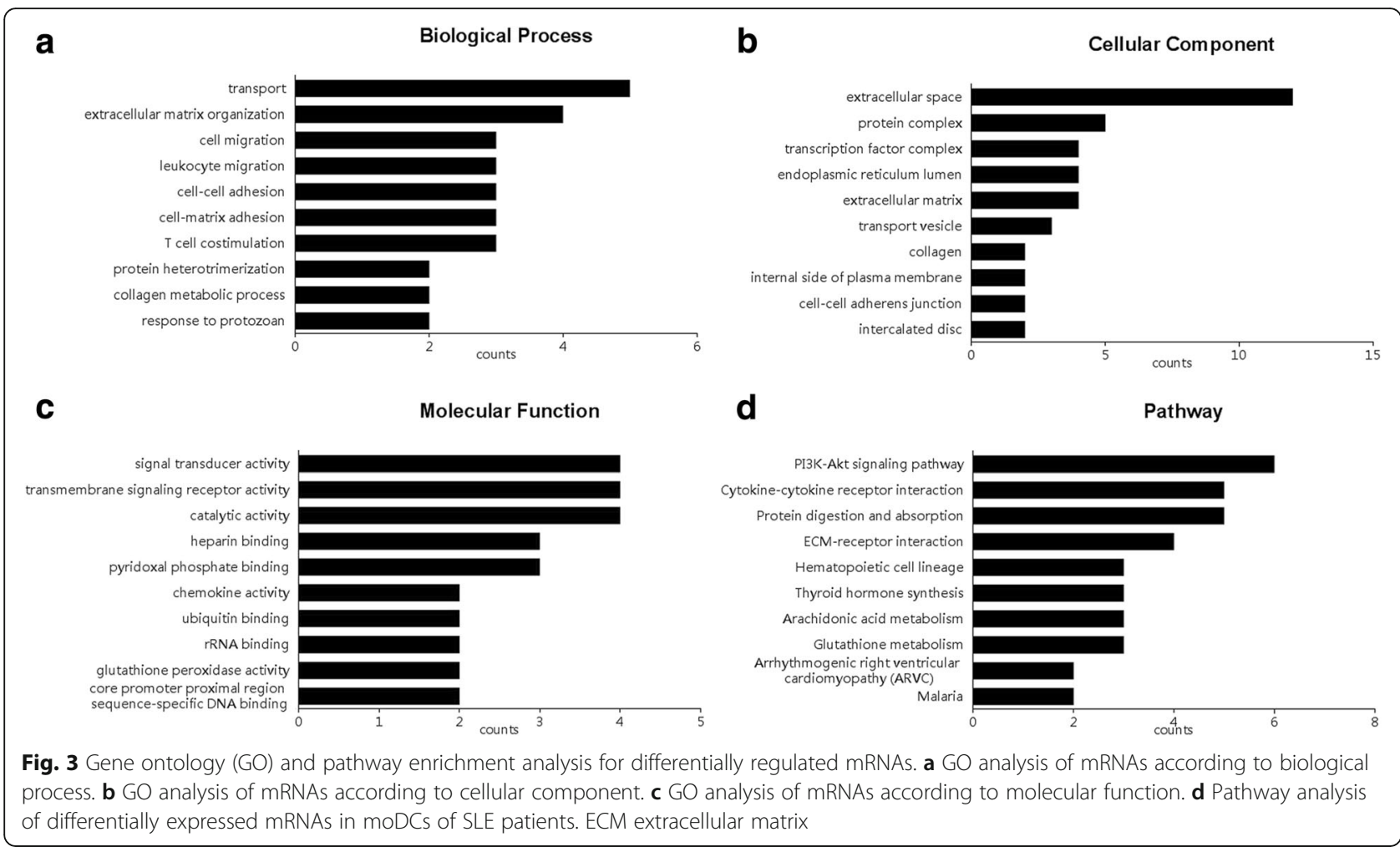

\section{Analysis of IncRNA-target gene regulatory network}

We identified the chromosomal coexpression genes 10 kbp upstream and downstream of the differentially expressed lncRNAs to determine potential lncRNA "cis" genes. The results of the "cis" analyses are shown in Additional file 4. (Table S3). Then we predicted the trans-associated genes of the differentially expressed IncRNAs with RNAplex v0.2, and the results are shown in Additional file 5 (Table S4). Matured moDCs could secrete an abundant source of both inflammatory and lymphoid chemokines, sustaining interaction of naive and activated $\mathrm{T}$ cells with antigen-presenting matured moDCs. We then constructed lncRNA-target genes of cytokines and chemokines using Cytoscape software (Additional file 6: Figure S2). Our data show that the lncRNA-target gene network was composed of 83 network nodes and 318 connections between 55 lncRNAs and 28 mRNAs. Within this coexpression network, CXCL10 was the most chemokine connected by lncRNAs and genes and IL- 6 was the most cytokine connected by lncRNAs and genes. Lnc-BSPH1-1:1, NR_024214, and ENST00000501122.2 were the most connected lncRNAs.

Confirmation of differentially expressed IncRNAs by qRT-PCR To confirm the reliability of the microarray data and considering the fold changes and the potential target genes of lncRNAs, we enlarged the sample sizes and selected five differentially expressed lncRNAs (three upregulated and two downregulated) and measured their expression level using qRT-PCR among 15 patients and 15 healthy controls including those 5 patients and 5 normal controls in the microarray. Consistent with the microarray data, the expression level of ENST00000604411.1 and ENST00000501122.2 were upregulated, and the expression level of lnc-HSFY2-3:3 and lnc-SERPINB9-1:2 were downregulated. However, the expression level of ENST00000568394.1 showed the same changed pattern as seen in the microarray analysis with no statistical significance (Fig. 4).

\section{Correlation between the aberrantly expressed IncRNAs and SLEDAI score of patients with SLE}

We further analyzed the correlation between the expression levels of the lncRNAs ENST00000604411.1, ENST00000501122.2, lnc-HSFY2-3:3, lnc-SERPINB9-1:2 and SLEDAI score. The expression levels of ENST00000604411.1 $(r=0.593, \quad P=0.020)$ and ENST00000501122.2 $(r=0.539, P=0.038)$ were positively correlated with the SLEDAI score. However, neither lnc-HSFY2-3:3 nor lnc-SERPINB9-1:2 was significantly correlated with SLEDAI score (Fig. 5).

\section{Discussion}

Long noncoding RNAs, more than 200 nucleotides in length, are nonprotein-coding transcripts with a lack of an open reading frame. They can regulate gene expression at 

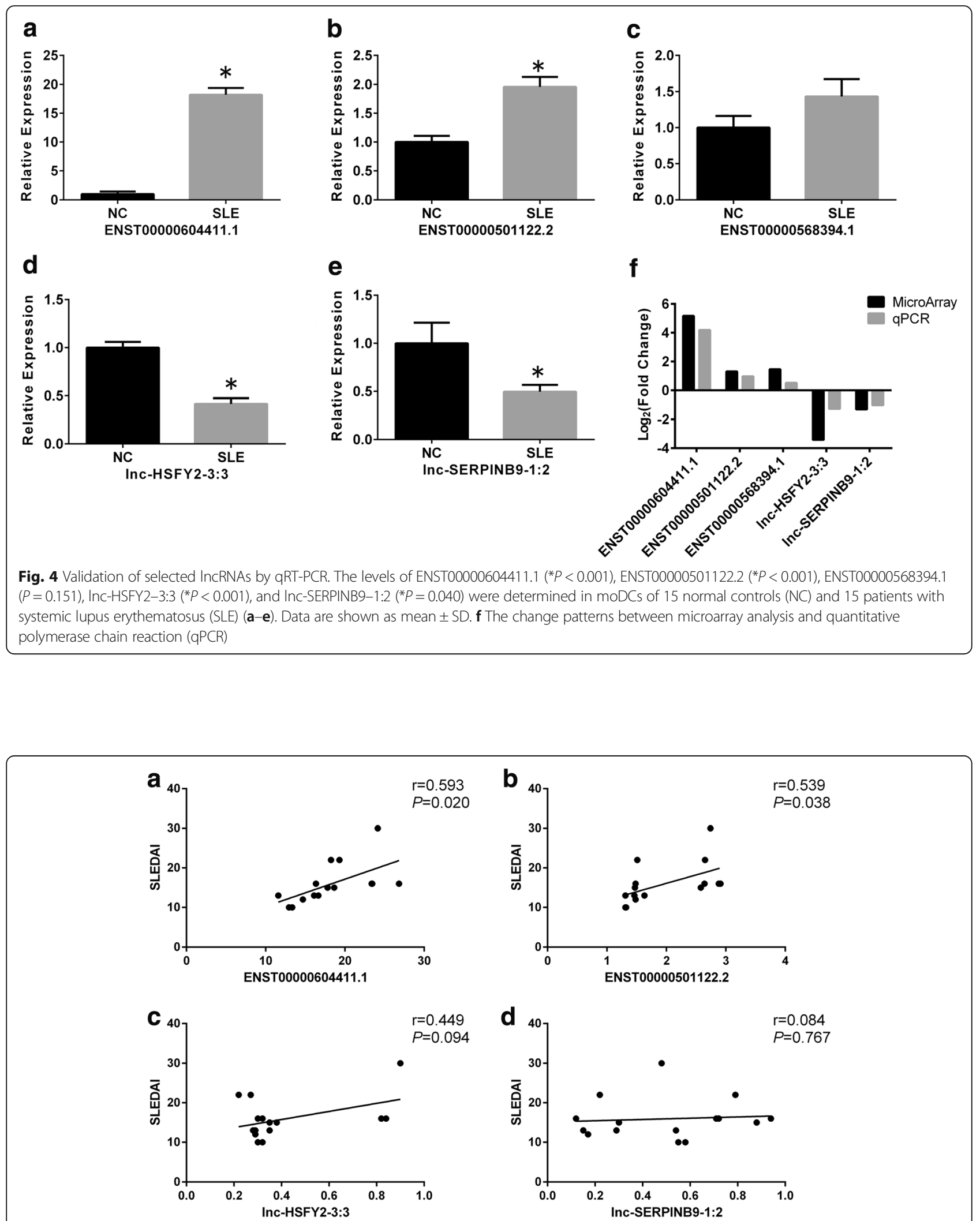

Fig. 5 Correlation between IncRNAs and systemic lupus erythematosus disease activity index (SLEDAI). a, b ENST00000604411.1 and ENST00000501122.2 expression was positively correlated with SLEDAI score. $\mathbf{c}$ No significantly correlation was observed between Inc-HSFY2-3:3 and SLEDAI score. $\mathbf{d}$ No significantly correlation was observed between Inc-SERPINB9-1:2 and SLEDAI score 
the level of chromatin remodeling, gene transcription, protein transport, and trafficking [14]. There is increasing interest in the potential involvement of lncRNAs in a number of complex human diseases, including autoimmune diseases, neurological disorders, coronary artery disease, and various cancers [11, 14, 15]. Genetic evidence suggests that IncRNA GAS5, a prime candidate for the chromosome 1q25 SLE locus, is related to susceptibility for SLE [16]. GAS5 also has been linked with an increased susceptibility to SLE in mouse models, presumably as a result of its effect on the immunosuppressant role of glucocorticoids [17]. The increased IncRNA NEAT1 expression in monocytes is related to the elevated production of a number of cytokines and chemokines in SLE patients [18]. LncRNA MALAT-1 expression was abnormally increased in monocytes of SLE patients, and silencing MALAT-1 significantly reduced the expression of IL-21 in primary monocytes of SLE patients by regulating SIRT1 signaling [19]. This all suggests that lncRNAs could contribute to the pathogenesis of SLE. Furthermore, lncRNAs could also serve as potential biomarkers in SLE. For instance, Linc0949 is decreased in PBMCs of patients with SLE. It can significantly increase following effective treatment for lupus, suggesting its potential as a biomarker for diagnosis, disease activity, and therapeutic response in SLE [20]. GAS5, linc0597, and lnc-DC in plasma may also specifically identify patients with SLE [21].

Published studies involving aberrantly expressed IncRNAs in SLE patients have mainly focused on PBMCs [20, 22], T cells [23], monocytes [18], and plasma [21]. However, there are no current studies regarding lncRNAs of DCs in SLE patients. Since lncRNAs appear to be expressed in a much more cell type-specific manner than transcription factors and other protein-coding genes, the aim of our study was to explore aberrant lncRNA expression in moDCs of SLE patients to provide new insight into the pathogenesis of SLE.

We analyzed five moDC samples from individual SLE patients and five moDC samples from normal controls using lncRNA and mRNA microarrays. Based on the microarray data, we found 163 lncRNAs and 137 mRNAs that were differentially expressed. GO and KEGG pathway analyses showed that the differentially expressed mRNAs on moDCs mainly related to T-cell costimulation, chemokine activity, and cytokine-cytokine receptor interaction that are clearly associated with SLE pathogenesis.

We used qRT-PCR to validate the lncRNA microarray results in 15 patients and 15 normal controls, including those in the microarrays. Based on the qRT-PCR results, ENST00000604411.1, ENST00000501122.2, lnc-HSFY23:3, and lnc-SERPINB9-1:2 were differentially expressed, which was in agreement with the microarray results. The expression level of ENST00000568394.1 showed the same change pattern as shown in the microarray analysis with no statistical significance, which is likely due to the fact that the expanded test sample size for the qRT-PCR might have excluded some of the false positive results obtained in the microarray or due to technical limitations, such as cross-hybridization, signal saturation, and limited dynamic range in the microarray.

ENST00000604411.1, known as TSIX or LINC00013, expresses a noncoding antisense transcript across the 3' end of the XIST locus. TSIX was overexpressed in systemic sclerosis ( $\mathrm{SSc}$ ) dermal fibroblasts both in vivo and in vitro, and is higher in SSc sera. TSIX is a new regulator of collagen expression which stabilizes the collagen mRNA [24]. It also protects the active-X from ectopic silencing once X-inactivation has commenced [25]. There is an increased incidence and prevalence of systemic lupus erythematosus in females, which might involve $\mathrm{X}$ chromosome inactivation [26]. In our study, we found ENST00000604411.1 was increased in moDCs of SLE patients and the expression level of ENST00000604411.1 was positively correlated with the SLEDAI score. Therefore, the upregulated ENST00000604411.1 might facilitate $\mathrm{X}$ chromosome inactivation through protecting the active- $\mathrm{X}$ from ectopic silencing and take part in the pathogenesis of SLE; however, further studies need to be performed to know exactly what role TSIX plays in the processes of the disease.

ENST00000501122.2 is a 22.74-kb intergenic lncRNA transcript, known as NEAT1. This lncRNA is retained in the nucleus where it forms the core structural component of the paraspeckle suborganelles. Zhang et al. [27] found NEAT1 expression was abnormally increased in SLE patients and predominantly expressed in human monocytes. There was also a positive correlation between NEAT1 and clinical disease activity in SLE patients. Furthermore, silencing NEAT1 significantly reduced the expression of a group of chemokines and cytokines, including IL-6, CXCL10, etc., which were induced by LPS continuously and in late stages [27]. NEAT1 is also critical for the expression of IL-8 [28]. In our study, we found NEAT1 expression was increased in moDCs from SLE patients. Therefore, NEAT1 was upregulated in both moDCs and their parent monocytes in SLE. We have also previously found IL-6 expression was increased in moDCs of SLE patients [8]. Future studies should focus on whether the increased NEAT1 expression in moDCs impact the cells to produce increased cytokines and chemokines in SLE patients.

Widespread change in IncRNAs might regulate the gene expression and production of inflammatory mediators in moDCs. Lnc-DC knockdown impacted the antigen uptake function of moDCs, impaired allogenic $\mathrm{CD} 4^{+}$ T-cell proliferation, and reduced the strength of cytokine release [12]. A previous study demonstrated that 
lincRNA-Cox2, a critical inflammation mediator, was induced in bone marrow-derived DCs after stimulation with LPS [29]. A further study revealed that lincRNA-Cox2 mediated both the activation and repression of distinct classes of immune genes, including Irf7, CCL5, and IL-6, etc. [30]. Moreover, lncRNAs, such as THRIL, PACER, and lincRNA-EPS, can also regulate the inducible expression of cytokines following immune activation [31]. In the current study, we found that the predicted target genes of differentially expressed lncRNAs in moDCs included cytokines and chemokines, especially IL-6, CXCL10, IL-10, CXCL2, etc. We also observed that the differentially expressed mRNAs in moDCs of SLE patients were enriched in the process of cell migration and chemokine activity and in the pathways of cytokine-cytokine receptor interaction. This is similar to our previous observation that moDCs in SLE manifested proinflammatory functions such as producing elevated levels of IL-6, CCL2, and CCL5, with the attraction of more $\mathrm{CD} 4^{+} \mathrm{T}$ cells compared with moDCs of healthy controls [8]. In addition, the majority of patients with SLE display an increased expression of type I interferon (IFN)-regulated genes, also known as an IFN signature [32]. We found that some target genes of the differentially expressed IncRNAs in moDCs are connected to the type I IFN system, such as IRF5 and TREX1. IRF5, the target gene of IncRNA NR_034053.2, is associated with increased serum IFN activity in SLE patients [33]. The target gene of lncRNA n339353 is TREX1, and loss-of-function mutations in this leads to accumulation of intracellular DNA that triggers type I IFN production [34]. The expression level of both lncRNA NR_034053.2 and lncRNA n339353 is elevated in moDCs of SLE patients, which might suggest a regulatory role of moDCs in producing type I IFN in SLE. Since cytokine production is one of the major functions of DCs with great biological importance, more studies are needed to focus on the crosstalk between cytokines and lncRNAs in DCs; the functions of these differentially expressed lncRNAs require further study.

The limitation of our study is that we have not examined lncRNA profiles in circulating myeloid DCs (mDC) and plasmacytoid DCs (pDC) in SLE patients, which is more meaningful in the clinic. However, since the population of $\mathrm{mDC}$ and $\mathrm{pDC}$ is very small in the peripheral blood, it is difficult to obtain enough cells for study. We therefore utilized the well-accepted model of human DC differentiation from peripheral blood monocytes under inflammatory conditions [12], and we have successfully identified those cells as DCs according to their morphology, phenotype, and function. The potential disadvantage of using moDCs is that it is an artificial system and could be affected by external environments. However, moDCs in our study were differentiated under the same conditions. As a result, the discrepancy between the SLE group and the healthy control group might be due to the intrinsic factors. Another major limitation is that all SLE patients and healthy controls in our study are Chinese females, and so there is gender bias in our findings. Our results may not reflect to male patients and patients of other ethnic backgrounds. Since DCs are the master regulators for initiation, amplification, and perpetuation of SLE [35], targeting DCs may be of benefit for treatment in the future.

\section{Conclusions}

Our study provides comprehensive lncRNA and mRNA profiles for moDCs in SLE patients. The differential expression of ENST00000604411.1, ENST00000501122.2, lnc-HSFY2-3:3, and lnc-SERPINB9-1:2 may participate in the pathogenesis of SLE. The expression level of ENST00000604411.1 and ENST00000501122.2 was positively correlated with SLEDAI score and may have disease activity evaluation value for SLE.

\section{Additional files}

Additional file 1: Table S1. The specific information about the differentially expressed IncRNAs. (XLSX $76 \mathrm{~kb}$ )

Additional file 2: Table S2. The specific information about the differentially expressed mRNAs. (XLSX 83 kb)

Additional file 3: Figure S1. Coexpression network with differentially expressed IncRNAs and mRNAs. Red triangles represent upregulated IncRNA, green triangles represent downregulated IncRNA, circles with a yellow border represent upregulated mRNA, and circles with a blue border represent downregulated mRNA. Arrows represent positive correlation and terminated lines represent negative correlation. (PDF $33 \mathrm{~kb}$ )

Additional file 4: Table S3. The predicted cis-associated genes of the differentially expressed IncRNAs. (XLSX $2714 \mathrm{~kb}$ )

Additional file 5: Table S4. The predicted trans-associated genes of the differentially expressed IncRNAs. (XLSX 7838 kb)

Additional file 6: Figure S2. LncRNAs and targeted genes regulating cytokine and chemokine networks. The node color differs from dark green to dark red according to the connection numbers from small to large. Squares represent target genes. Circles represent IncRNAs. Arrows represent positive correlation and terminated lines represent negative correlation. (PDF $32 \mathrm{~kb}$ )

\section{Abbreviations}

APC: Antigen-presenting cell; DC: Dendritic cell; FDR: False discovery rate; GAPDH: Glyceraldehyde 3-phosphate dehydrogenase; GO: Gene ontology; IFN: Interferon; IL: Interleukin; KEGG: Kyoto Encyclopedia of Genes and Genomes; IncRNA: Long noncoding RNA; LPS: Lipopolysaccharide; moDC: Monocyte-derived dendritic cell; PBMC: Peripheral blood mononuclear cell; qRT-PCR: Quantitative real-time polymerase chain reaction; SLE: Systemic lupus erythematosus; SLEDAI: Systemic lupus erythematosus disease activity index; SSc: Systemic sclerosis

\section{Funding}

This research was supported by the National Natural Science Foundation of China (grant no. 81703122, 81773324) and the Natural Science Foundation of Shanghai (16ZR1404900). 


\section{Availability of data and materials}

The microarray data have been deposited in Gene Expression Omnibus (GEO) under accession number GSE89240.

\section{Authors' contributions}

YW cultured the cells, performed the microarray, and drafted the manuscript. ShC and SuC participated in the design of the study and contributed to the analyses of the results. JD participated in the qPCR experiments. JL and HQ participated in study design and target gene prediction. JW contributed to data interpretation and manuscript revision. JL and JX conceived the study, participated in its design and coordination, and revised the manuscript. All authors read and approved the final version to be published.

\section{Ethics approval and consent to participate}

The study was approved by the Independent Ethics Committee of Huashan Hospital and written informed consent was obtained from all subjects.

\section{Consent for publication}

Written informed consents were obtained from the patients for publication of their individual details and accompanying images in this manuscript. The consent form is held by the authors and is available for review by the Editor-in-Chief.

\section{Competing interests}

The authors declare that they have no competing interests.

\section{Publisher's Note}

Springer Nature remains neutral with regard to jurisdictional claims in published maps and institutional affiliations.

\section{Author details \\ ${ }^{1}$ Department of Dermatology, Huashan Hospital, Fudan University, 12 Wulumuqi Zhong Road, Shanghai 200040, People's Republic of China ${ }^{2}$ Department of Human Anatomy and Histoembryology, School of Basic Medical Science, Fudan University, Shanghai, People's Republic of China.}

Received: 19 March 2018 Accepted: 1 June 2018

\section{Published online: 11 July 2018}

\section{References}

1. Mackern-Oberti JP, Llanos C, Riedel CA, Bueno SM, Kalergis AM. Contribution of dendritic cells to the autoimmune pathology of systemic lupus erythematosus. Immunology. 2015;146(4):497-507.

2. Mahajan A, Herrmann M, Munoz LE. Clearance deficiency and cell death pathways: a model for the pathogenesis of SLE. Front Immunol. 2016;7:1-12.

3. Klarquist J, Zhou Z, Shen N, Janssen EM. Dendritic cells in systemic lupus erythematosus: from pathogenic players to therapeutic tools. Mediat Inflamm. 2016;2016:5045248.

4. Son M, Kim SJ, Diamond B. SLE-associated risk factors affect DC function. Immunol Rev. 2016;269(1):100-17.

5. Chan VS, Nie YJ, Shen N, Yan S, Mok MY, Lau CS. Distinct roles of myeloid and plasmacytoid dendritic cells in systemic lupus erythematosus. Autoimmun Rev. 2012;11(12):890-7.

6. Ding D, Mehta H, McCune WJ, Kaplan MJ. Aberrant phenotype and function of myeloid dendritic cells in systemic lupus erythematosus. J Immunol. 2006;177(9):5878-89.

7. Estrada-Capetillo L, Hernandez-Castro B, Monsivais-Urenda A, Alvarez-Quiroga C, Layseca-Espinosa E, Abud-Mendoza C, Baranda L, Urzainqui A, SanchezMadrid F, Gonzalez-Amaro R. Induction of Th17 lymphocytes and Treg cells by monocyte-derived dendritic cells in patients with rheumatoid arthritis and systemic lupus erythematosus. Clin Dev Immunol. 2013;2013:584303.

8. Wang Y, Liang J, Qin H, Ge Y, Du J, Lin J, Zhu X, Wang J, Xu J. Elevated expression of miR-142-3p is related to the pro-inflammatory function of monocyte-derived dendritic cells in SLE. Arthritis Res Ther. 2016;18(1):263.

9. Wu HJ, Zhao M, Yoshimura A, Chang C, Lu QJ. Critical link between epigenetics and transcription factors in the induction of autoimmunity: a comprehensive review. Clinical Reviews in Allergy \& Immunology. 2016;50(3):333-44.

10. Atianand MK, Caffrey DR, Fitzgerald KA. Immunobiology of long noncoding RNAs. Annu Rev Immunol. 2017;35:177-98.
11. Faghihi MA, Modarresi F, Khalil AM, Wood DE, Sahagan BG, Morgan TE, Finch CE, Laurent GS, Kenny PJ, Wahlestedt C. Expression of a noncoding RNA is elevated in Alzheimer's disease and drives rapid feed-forward regulation of beta-secretase. Nat Med. 2008;14(7):723-30.

12. Wang $P$, Xue Y, Han Y, Lin L, Wu C, Xu S, Jiang Z, Xu J, Liu Q, Cao X. The STAT3-binding long noncoding RNA Inc-DC controls human dendritic cell differentiation. Science. 2014;344(6181):310-3.

13. Xin JX, Li J, Feng Y, Wang LY, Zhang Y, Yang RC. Downregulation of long noncoding RNA HOTAIRM1 promotes monocyte/dendritic cell differentiation through competitively binding to endogenous miR-3960. Oncotargets Ther. 2017;10:1-9.

14. Li J, Xuan Z, Liu C. Long non-coding RNAs and complex human diseases. Int J Mol Sci. 2013;14(9):18790-808.

15. Gupta RA, Shah N, Wang KC, Kim J, Horlings HM, Wong DJ, Tsai MC, Hung $T$, Argani $P$, Rinn $J$, et al. Long non-coding RNA HOTAIR reprograms chromatin state to promote cancer metastasis. Nature. 2010:464(7291):1071-U1148.

16. Kino T, Hurt DE, Ichijo T, Nader N, Chrousos GP. Noncoding RNA Gas5 is a growth arrest- and starvation-associated repressor of the glucocorticoid receptor. Sci Signal. 2010;3(107):ra8.

17. Suarez-Gestal M, Calaza M, Endreffy E, Pullmann R, Ordi-Ros J, Sebastiani GD, Ruzickova S, Santos MJ, Papasteriades C, Marchini M, et al. Replication of recently identified systemic lupus erythematosus genetic associations: a case-control study. Arthritis Res Ther. 2009;11(3):R69.

18. Zhang FF, Wu LL, Qian J, Qu B, Xia SW, La T, Wu YF, Ma JY, Zeng J, Guo Q, et al. Identification of the long noncoding RNA NEAT1 as a novel inflammatory regulator acting through MAPK pathway in human lupus. J Autoimmun. 2016;75:96-104

19. Yang HX, Liang NX, Wang M, Fei YY, Sun J, Li ZY, Xu Y, Guo C, Cao ZL, Li $S Q$, et al. Long noncoding RNA MALAT-1 is a novel inflammatory regulator in human systemic lupus erythematosus. Oncotarget. 2017;8(44):77400-6.

20. Wu Y, Zhang F, Ma J, Zhang X, Wu L, Qu B, Xia S, Chen S, Tang Y, Shen N. Association of large intergenic noncoding RNA expression with disease activity and organ damage in systemic lupus erythematosus. Arthritis Res Ther. 2015;17:131.

21. Wu GC, Li J, Leng RX, Li XP, Li XM, Wang DG, Pan HF, Ye DQ. Identification of long non-coding RNAs GAS5, linc0597 and Inc-DC in plasma as novel biomarkers for systemic lupus erythematosus. Oncotarget. 2017;8(14):23650-63.

22. Luo Q, Li X, Xu C, Zeng L, Ye J, Guo Y, Huang Z, Li J. Integrative analysis of long non-coding RNAs and messenger RNA expression profiles in systemic lupus erythematosus. Mol Med Rep. 2018;17(3):3489-96.

23. Li LJ, Zhao W, Tao SS, Li J, Xu SZ, Wang JB, Leng RX, Fan YG, Pan HF, Ye DQ. Comprehensive long non-coding RNA expression profiling reveals their potential roles in systemic lupus erythematosus. Cell Immunol. 2017;319:17-27

24. Wang ZZ, Jinnin M, Nakamura K, Harada M, Kudo H, Nakayama W, Inoue K, Nakashima T, Honda N, Fukushima S, et al. Long non-coding RNA TSIX is upregulated in scleroderma dermal fibroblasts and controls collagen mRNA stabilization. Exp Dermatol. 2016;25(2):131-6.

25. Gayen S, Maclary E, Buttigieg E, Hinten M, Kalantry S. A primary role for the Tsix IncRNA in maintaining random X-chromosome inactivation. Cell Rep. 2015:11(8):1251-65.

26. McCombe PA, Greer JM, Mackay IR. Sexual dimorphism in autoimmune disease. Curr Mol Med. 2009;9(9):1058-79.

27. Zhang F, Wu L, Qian J, Qu B, Xia S, La T, Wu Y, Ma J, Zeng J, Guo Q et al. Identification of the long noncoding RNA NEAT1 as a novel inflammatory regulator acting through MAPK pathway in human lupus. J Autoimmun. 2016:75:96-104.

28. Imamura K, Imamachi N, Akizuki G, Kumakura M, Kawaguchi A, Nagata K, Kato A, Kawaguchi $Y$, Sato $H$, Yoneda $M$, et al. Long noncoding RNA NEAT1dependent SFPQ relocation from promoter region to Paraspeckle mediates IL8 expression upon immune stimuli. Mol Cell. 2014;53(3):393-406.

29. Guttman M, Amit I, Garber M, French C, Lin MF, Feldser D, Huarte M, Zuk O, Carey BW, Cassady JP, et al. Chromatin signature reveals over a thousand highly conserved large non-coding RNAs in mammals. Nature. 2009; 458(7235):223-7

30. Carpenter S, Aiello D, Atianand MK, Ricci EP, Gandhi P, Hall LL, Byron M, Monks B, Henry-Bezy M, Lawrence JB, et al. A long noncoding RNA mediates both activation and repression of immune response genes. Science. 2013;341(6147):789-92. 
31. Carpenter S, Fitzgerald KA. Cytokines and long noncoding RNAs. Cold Spring Harb Perspect Biol. 2018;10(6)

32. Bengtsson AA, Ronnblom L. Role of interferons in SLE. Best Pract Res Clin Rheumatol. 2017;31(3):415-28.

33. Niewold TB, Kelly JA, Flesch MH, Espinoza LR, Harley JB, Crow MK. Association of the IRF5 risk haplotype with high serum interferon-alpha activity in systemic lupus erythematosus patients. Arthritis Rheum. 2008;58(8):2481-7.

34. Lee-Kirsch MA, Gong M, Chowdhury D, Senenko L, Engel K, Lee YA, de Silva U, Bailey SL, Witte T, Vyse TJ, et al. Mutations in the gene encoding the 3'-5' DNA exonuclease TREX1 are associated with systemic lupus erythematosus. Nat Genet. 2007;39(9):1065-7.

35. Chan VSF, Nie YJ, Shen N, Yan S, Mok MY, Lau CS. Distinct roles of myeloid and plasmacytoid dendritic cells in systemic lupus erythematosus. Autoimmun Rev. 2012;11(12):890-7.

Ready to submit your research? Choose BMC and benefit from:

- fast, convenient online submission

- thorough peer review by experienced researchers in your field

- rapid publication on acceptance

- support for research data, including large and complex data types

- gold Open Access which fosters wider collaboration and increased citations

- maximum visibility for your research: over $100 \mathrm{M}$ website views per year

At BMC, research is always in progress.

Learn more biomedcentral.com/submissions 